\title{
Substance misuse in the severely mentally ill
}

\section{Roch Cantwell \& Glynn Harrison}

Comorbidity and dual diagnosis have a fashionable, and thus ephemeral, ring that belies their relevance to day-to-day practice. The topic has been increasingly addressed in North American literature where there is a recognition of the extent of substance misuse in the severely mentally ill, and the need to find ways of effectively managing its consequences. Substance misuse may colour the diagnosis, management and prognosis of major mental illness and can adversely affect the relationship between staff and patients. Despite its common occurrence, it frequently remains undetected (Ananth et al, 1989). With burgeoning research interest, there is some consensus as to how to manage the problem, but, as yet, little agreement on the precise nature of causal relationships.

\section{Links between substance misuse and psychosis}

The role of some substances of abuse in precipitating psychotic symptoms, usually in the context of intoxication or withdrawal states, is undisputed. Hallucinogen intoxication, almost by definition, is manifested by sensory distortion, illusion and hallucination. Stimulant use is frequently accompanied by paranoid symptoms. Such symptoms may also accompany the acute use of cannabis, cocaine and methylenedioxymethamphetamine (MDMA, 'Ecstasy'). Discontinuance syndromes are at their most dramatic in delirium tremens, but similar states can result from barbiturate and benzodiazepine withdrawal.

\section{Drug-induced psychosis}

The existence of prolonged or independent psychotic states triggered by substance use is more controversial. Alcoholic hallucinosis, while frequently described, remains perplexing (Glass, 1989). It may occur during heavy drinking and does not necessarily resolve on stopping, although abstinence is usually regarded as important in its management. The natural history is usually one of repeated relapses though these may take a variety of forms, including affective disorder, schizophrenia, and further episodes of hallucinosis, calling into question the nature of the initial illness. There may be evidence of cognitive impairment. The relationship to schizophrenia and delirium tremens is unclear, but treatment is conventionally with neuroleptics. Paranoid psychoses resulting from amphetamine use usually resolve within days to weeks of removal of the offending drug, often encouraged by antipsychotic medication. Cocaine psychoses appear to follow a similar path. Psychotic symptoms occurring in the course of prolonged hallucinogen use may also resolve over days to weeks, but characteristically, acute and transient 'flashbacks' occur, sometimes years after drug use has ceased.

\section{Cannabis and psychosis}

The greatest controversy reigns over the relationship between cannabis use and psychosis. Acute psychotic reactions occur but, despite many reports, there appears little conclusive evidence for a prolonged 'cannabis psychosis', and nojustification

Roch Cantwell, MRCPsych, Lecturer in Psychiatry, University of Nottingham, has carried out research in substance misuse and is currently investigating the prevalence of comorbidity in an inception cohort with psychotic disorders. Glynn Harrison, MRCPsych, Professor of Community Mental Health, Department of Psychiatry, University of Nottingham, has a special interest in the epidemiology of schizophrenia, including long-term course and outcome, and migration. 
for its use as a diagnostic label. Cannabis has been cited as an independent risk factor in the development of schizophrenia, the strongest evidence coming from a prospective study of Swedish conscripts followed up over a 15 year period (Andreasson et al, 1987). The relative risk of developing schizophrenia was 2.4 for cannabis users, and 6.0 for heavy users, when compared with non-users at conscription. The study, and a further related paper, have been criticised on methodological grounds (Thomas, 1993), not least because of the difficulty in establishing causal relationships. Nevertheless, it has been reported that relatives of schizophrenic patients who abuse cannabis have themselves an increased risk of schizophrenia, suggesting a gene to environment (cannabis) effect in certain individuals (McGuire et al, 1995). While any aetiological links between cannabis and schizophrenia remain uncertain, the detrimental effects of cannabis use on the course of pre-existing illness are more clearly established.

\section{Extent of dual diagnosis}

\section{Prevalence issues}

The prevalence of comorbidity is not easy to assess. Rates quoted range from 15-65\%. Accurate case definition is absent from earlier studies, and there is great variety in the choice of setting (in/outpatient, general population) and in subjects (primary substance misusers, severely mentally ill). Extrapolating work carried out in North America to Europe is difficult given that substance misuse is greatly influenced by demographic factors, and by temporal fluctuations. Finally, most studies have concentrated on patients with chronic illness, confounding any attempts to identify aetiological links between substance use and psychosis. The questions which need to be addressed by epidemiological studies, and which are important for clinical practice, include whether substance misuse is more common in severe mental illness than in the general population, whether rates of comorbidity differ between specific psychotic disorders, and whether certain substances are preferentially used over others.

The Epidemiological Catchment Area (ECA) study revealed a prevalence of $47 \%$ and $32 \%$ for substance misuse in schizophrenia and affective disorders respectively (Regier et al, 1990). Schizophrenic patients were 4.6 times more likely, and affective disordered patients 2.6 times more likely, to have drug or alcohol problems when compared with the general population. A further review of several studies suggests an increase over time in the prevalence of comorbidity (Cuffel, 1992). Whether substance misuse is more common in certain types of severe mental illnesses is less easily determined. An increase among patients with schizophrenia is strongly suggested by the ECA study, where only those with antisocial personality showed higher rates of substance use. There is a trend toward increased alcohol use in bipolar disorder, and recent evidence from first onset psychosis studies also found a raised prevalence in those with affective illness (e.g. Strakowski et al, 1993). Overall, however, those most at risk of drug and alcohol comorbidity remain young men with chronic psychotic illness.

\section{Preferential use}

Those with chronic illness appear to preferentially use certain substances over others. Hallucinogens and stimulants are more often used, and central nervous system depressants such as alcohol, hypnotics and opiates, less used in schizophrenia (Schneier \& Siris, 1987). While this might be expected if a causal link between psychostimulants and psychosis is assumed, patients continue to use these drugs often in the clear knowledge, and experience, of the detrimental effect on their mental state.

\section{Reasons for substance use}

Psychotic patients may be at risk of substance misuse for a number of reasons. Downward social drift, associated especially with schizophrenia and the shift from institutional care, increase exposure of patients to drugs of abuse (Smith \& Hucker, 1994). At the same time, there has been a growing acceptability and availability of drug use in society. Patients with severe mental illness may be least able to resist peer pressure toward drug use, and, as a result of their illness, they share many of the risk factors (negative affective states, poor self-esteem and role performance, impaired social skills and social isolation) associated with drug use in the general population (Lehman et al, 1989). The knowledge that psychotic symptoms can be reproduced by a drug may impart a sense of control to patients, increasing use despite their recognition of the adverse effects (Selzer \& Lieberman, 1993). Reasons given by patients for their drug use, such as the relief of boredom, decrease in anxiety and improved socialisation, do not differ much from 
those of non-psychotic users, but the mentally ill often experience these difficulties more acutely than other drug users (Box 1).

\section{Premorbid functioning}

An intriguing finding from several studies has been the evidence for better premorbid functioning in schizophrenic patients with substance misuse (e.g. Arndt et al, 1992). It may be that those with greater social skills in the premorbid period are more likely to come in contact with drug and alcohol use, and that following the onset of illness, are then at greater risk of escalating use. Paradoxically then, those patients who might be regarded as having the best prognosis may be most at risk of descending into drug use, with its attendant problems.

\section{Self-medication}

Patients may use alcohol and drugs to 'selfmedicate' either their symptoms (whether positive, negative or dysphoric) or the side-effects of psychotropic medications. Alcohol and opiates can lessen the distress associated with positive psychotic symptoms, while psychostimulants such as amphetamines and cocaine are reported to counteract negative symptoms. Cannabis may also attenuate apathy and withdrawal - higher rates of negative symptoms are reported among non-users. The action of opiates in masking positive symptoms may delay the onset or diagnosis of psychosis, and it has been suggested that low rates of opiate abuse in schizophrenia could be explained by a powerful self-medicating action preventing presentation. It seems more likely, however, that the reduced cooccurrence relates to the socialisation and motivation often lacking in chronic patients, but which is essential to maintain an intravenous drug habit. Depressive symptoms have been found to be more prevalent in cocaine users, suggesting either that the drug worsens dysphoria or that it may be used

Box 1. Reasons for substance use in severe mental illness

Move from institutional to community care

Downward social drift

General increase in use and acceptability

Facilitate socialisation, relieve boredom

Self-medication of positive, negative or dysphoric symptoms

Self-medication of antipsychotic sideeffects as self-medication in those who show a more depressed clinical picture.

Side-effects of neuroleptics can be particularly disturbing, and some have suggested that a rise in comorbidity has mirrored the introduction and increasing prescribing of these medications (Cuffel, 1992). In one in-patient sample, $15 \%$ of patients gave side-effects of medications as their reason for drug use (Dixon et al, 1991). Both alcohol and cannabis are cited as likely to ameliorate unwanted effects. Alcohol use has been related to subjective distress associated with akathisia, perhaps being used by patients to alleviate the accompanying agitation and dysphoria (Duke et al, 1994).

Comorbidity as a means of self-medication might be explained in terms of the neuropharmacological effects of drugs of abuse. Drugs with anticholinergic actions, such as cannabis, or dopaminergic effects, such as amphetamines, could be expected to ameliorate negative symptoms. Both cocaine and cannabis may exert their subjective beneficial effects by specifically antagonising the action of neuroleptics (Bowers et al, 1990).

\section{Implications of dual diagnosis}

Those that stand out as substance users among the severely mentally ill are young males. This is not surprising - it is also true in the general population. However, several studies suggest that substance users are not only younger but have an earlier age of onset of their psychosis. Breakey et al (1974) found an average difference of four years in onset of both symptoms and hospitalisation among their comorbid population when compared with uncomplicated schizophrenic subjects. Earlier onset might suggest a role for drugs as precipitants of psychosis in vulnerable individuals, advancing the onset of the disorder by several years. Attempts to examine premorbid vulnerability have tended to reveal dual diagnosis patients as a less impaired group when compared to non-abusing schizophrenics (Breakey et al, 1974; Arndt et al, 1992) and better premorbid functioning has also been used as evidence for a causal role for drugs in precipitating psychosis.

Good premorbid adjustment might suggest a better symptom profile and prognosis. Dixon et al (1991) found fewer positive and negative symptoms at discharge among a group of consecutively admitted patients with schizophrenia, schizoaffective disorder or schizophreniform disorder, and associated substance abuse or dependence. Other findings confirming this hypothesis include fewer hospitalisations among cocaine abusing patients with major mental illness, less negative 
symptoms in dual diagnosis patients at onset of psychosis, and better psychosocial functioning among a treatment-resistant comorbid group.

However, the overriding impression from studies examining psychopathology and prognosis in dually diagnosed subjects is that patients are adversely affected by their substance use (Box 2). Among those who use cannabis, use is associated with greater severity of psychotic symptoms, and earlier, and more frequent relapses (Linszen et al, 1994; Martinez-Arevalo et al, 1994). Cocaine and alcohol in turn are associated with more affective symptoms, and alcohol misuse has been linked to the development of tardive dyskinesia (Duke et al, 1994). A less favourable outcome in comorbidity was also confirmed at two years in the World Health Organization Determinants of Outcome of Severe Mental Disorders study, although poorer compliance with medication was a confounding factor in those patients (Jablensky et al, 1992).

Allowing for the confounding effects of brief 'drug induced' psychoses, the weight of evidence therefore points to poorer prognosis in those with established psychotic illness. This group is characterised by a greater frequency of hospitalisation and greater use of emergency services (Bartels et al, 1993). When not in hospital they are less able to cope with the rigors of daily life when compared with their non-misusing counterparts. They are more likely to be homeless and less effective in dealing with finances (Drake \& Wallach, 1989). They show a greater propensity for violent behaviour, both current and future (Cuffel et al, 1994), and for suicidality (Drake \& Wallach, 1989). Despite their increased hospitalisation, comorbid patients are more difficult to engage in treatment and comply poorly with medication. It is no surprise then that this group responds poorly to treatment, although this may be more than a function of their non-compliance. Bowers et al (1990) gave fixed doses of neuroleptics to psychotic subjects in the early stages of illness and found that those with a history of psychotogenic drug use had

Box 2. Complications of substance misuse

Exacerbation of symptoms

Increased relapse and rehospitalisation

Homelessness and downward social drift

Violent and criminal behaviour

Poor compliance

Decreased response to medication

Poor prognosis and outcome in established psychotic illness a poorer response. They suggest that drug use may contribute to relative neuroleptic refractoriness. A similar lessening of effectiveness of drug treatment has been reported for lithium in dual diagnosis mania (Black et al, 1988).

In summary, substance misusers who develop psychosis may appear to be a better functioning group, and, in the short term, even show an improved outcome. Continued use, however, predicts greater symptomatology, higher rates of relapse, more social impairment and poorer compliance with management. A two-stage model has been suggested to explain this dichotomy (Arndt et al, 1992). Initially, good premorbid adjustment and sociability increase the likelihood of exposure to drugs and alcohol. Subsequently, the development of psychotic symptoms accelerates use in an attempt to cope with the stress of mental illness. However, it may also be possible that studies have examined two separate groups. Those in the acute stages of illness may indeed have a milder disorder which has been exacerbated by substance use. Discontinuation of drug use and adequate treatment would lead to rapid resolution of symptoms and better prognosis. The chronically mentally ill, who drift into drug use because of social disadvantage and perhaps self-medication, are more disorganised by their use, and less equipped to escape from drug-using circles. Only longitudinal studies, following dual diagnosis patients in inception cohorts, can answer this question.

\section{Management}

Dual diagnosis patients are often at a disadvantage even before treatment begins. Their substance use frequently remains undetected, and thus unaddressed. They are more likely to drop out of management plans and comply poorly with medication. Management is not helped by the traditional structure of psychiatric services, which attempts to streamline patients along general or substance misuse pathways. Most clinicians will recognise the scenario where those presenting to one service with psychotic and substance misuse symptoms, are redirected when treatment fails or the other aspect of their diagnosis is relabelled as 'primary', only to return again at a later date (the 'ping-pong effect'). Staff may feel deskilled when only able to address one facet of the problem, and frustration with the almost inevitable lack of progress can lead to the rejection of a patient who is seen as 'attention-seeking and help-rejecting'. 
Patients' characteristics contribute to difficulties. Their disruptive behaviour, poor compliance, and greater propensity for violence may result in refusal of admission and premature discharge. Medication regimes may be compromised because of fears of drug interactions or self-harm, and Mental Health Act provisions underused when symptoms are judged to be induced by alcohol or drug taking.

The first principle of effective management is a high index of suspicion (Box 3). Careful information gathering can be supplemented by laboratory screening, which should probably be routine in all cases of psychosis at first presentation and, where appropriate, in unexplained relapses in the chronically ill. There is a danger, however, in overreliance on urine samples and blood tests. Many drugs of abuse remain detectable for only brief periods of time, and the sensitivity of most readily available liver function tests is inferior to the simple 'CAGE' questionnaire (Chick et al, 1993). Highest levels of detection are achieved if several approaches are combined - including information from the patient and carers, and laboratory investigations.

\section{Who should care for dual diagnosis patients?}

An agreed policy on who should manage dual diagnosis patients will help to avoid the 'ping-pong effect', and develop skills and confidence among staff. Most comparative research in this area has been carried out in North America, where substance misuse services are often designed around the Minnesota or '12-step' Alcoholics Anonymous model of treatment, with its emphasis on abstinence and confrontational groupwork. Not surprisingly, this has several drawbacks for those with psychotic illness, and workers have tended to suggest that dual diagnosis patients are best managed within general psychiatry services (Selzer \& Lieberman, 1993). The divisions are not so clearcut in a UK setting, where substance misuse services are usually more eclectic. The best solution may well be a dedicated 'dual diagnosis service', but at a minimum, close cooperation between substance misuse and general psychiatry, with policies on continuity of keyworkers and access to appropriate day and in-patient facilities if required, is essential.

\section{Integrated treatments}

Any management plan should be integrated, attempting to deal with both aspects of the problem, although gaining control of the substance misuse will often result in a lessening of psychotic

\section{Box 3. Good practice points}

High index of suspicion-substance misuse is frequently denied, and often not assessed. Use screening devices, such as urinalysis routinely

Integrated treatment - treat both aspects of the problem and avoid the 'ping-pong' effect

Social support - patients often require help with housing and finances. Addressing social problems may improve compliance

Education - about detrimental effects of alcohol and drugs, relapse prevention, and harm reduction

Long-term focus - change is slow and relapse usual rather than exceptional. It should not lead to discharge from care

Optimistic approach - patients need continued encouragement, especially during crises or relapse

Team support - these are difficult patients to manage. Staff need to acquire appropriate skills and confidence

symptoms as well. Because of the higher dropout rates from treatment in this group, the early stages of management should focus on engagement and building a therapeutic relationship. Insistence on abstinence at this stage is likely to result in dropout. To date, studies have shown flexible approaches to be more successful at keeping patients in treatment. Even when abstinence is achieved, carers may need to be reminded that relapse is the norm rather than the exception, and will not lead to exclusion from treatment. Dropouts will need assertive follow-up in the community, and the relevant provisions of the Mental Health Act used where appropriate. Management must also take into account the slow nature of progress. In one study, those who achieved stable abstinence from alcohol did so after an average of two years treatment (Drake et al, 1993). These patients are more socially disadvantaged. They tend to have few supports and are more likely to be homeless, unemployed, and to have poor control over their finances. Care plans which address social problems will be more successful in retaining patients in treatment.

In common with most substance misuse programmes, regular monitoring of drug and alcohol use is important, though the approach is less confrontational or challenging. Patients need education in relapse prevention and in relapse management. This can take the form of discussions 
on triggers to relapse, rehearsing how to deal with potentially difficult social situations, and altering daily routines to reduce risk. Because of more chaotic lifestyles, patients with comorbid illness may be at greater risk of complications of drug misuse, including HIV and other infections, and particular attention should be paid to harm reduction strategies. Often the greatest difficulty workers face is in balancing a quick response to patients' problems as they arise, with the development of a care plan which focuses on long-term goals and recognises the slow rate of progress. Good peer support and supervision is essential for staff. Continued encouragement and an optimistic approach by staff is essential for patients.

\section{Pharmacological management}

The choice of medication for dual diagnosis patients can cause anxiety. In treating any acute episode, it is important to attempt to delineate intoxication or withdrawal syndromes from exacerbation of the underlying psychosis. Neuroleptics are effective in treating psychotic reactions following drug use in patients without schizophrenia, so their use is justified in dual diagnosis patients, where the genesis of psychotic symptoms is seldom clear. Where drug use has contributed to acute relapse, it has been suggested that highpotency neuroleptics may be more advisable, given their lower risk in exacerbating substance-induced hypotension, tachycardia, and anticholinergic effects (Siris, 1990).

Even more care than usual may be needed in guarding against side-effects, as substance misuse may increase in order to self-medicate unwanted symptoms, such as neuroleptic-induced akathisia and akinesia. Anti-Parkinsonian drugs should not be withheld if side-effects require treatment, but their potential for abuse must be borne in mind. Depot neuroleptic preparations are sensible given the risk of poor compliance. Establishing a stable dose regime may not be easy. Chronic alcohol use, for example, causes hepatic enzyme induction, thus reducing neuroleptic bioavailability. Acute alcohol binges, however, may impair drug metabolism, resulting in increased drug effects (Salloum et al, 1991). The best compromise may be the use of depot preparations, with 'top-up' oral medications adjusted according to severity of symptoms and current substance use. Obviously, a good therapeutic relationship will make this easier. The role of newer antipsychotic medications is largely untested, but at least one report suggests they are no less effective in dual diagnosis patients than in uncomplicated schizophrenia (Buckley et al, 1994).
Depressive syndromes are common in schizophrenia and in many substance abuse disorders. If antidepressants are indicated, selective serotonin reuptake inhibitors may be more appropriate than tricyclic drugs, given the risk of overdose and interactions associated with the latter. However, apart from their relative safety in association with alcohol, little is known about interactions between SSRIs and other drugs of abuse. Monoamine oxidase inhibitors should be avoided because of the risk of hypertensive crises. Lithium can be used if clinically indicated, but is limited by the need for good compliance. Cases should be judged individually. Benzodiazepines are clearly appropriate in some withdrawal syndromes, but their use in managing insomnia or anxiety symptoms should be time-limited and cautious. They are prone to abuse and may also exacerbate behavioural disinhibition, increasing aggression and self-harm. Because of the fashion for intravenous abuse of temazepam, sometimes resulting in serious medical complications such as peripheral vascular occlusion and gangrene, this drug is best avoided.

It is worth keeping in mind that many psychotropic medications, including antipsychotics and antidepressants, can lower fit thresholds. While this is unlikely to be a reason for avoiding their use in dual diagnosis patients, extra caution may need to be exercised in alcohol, barbiturate and benzodiazepine withdrawal states. Often, an upward adjustment or slower reduction in benzodiazepine treatment regimes will suffice.

Pharmacological treatments for substance misuse may also have potential for interactions. Disulfiram, through its inhibition of dopamine beta-hydroxylase, has been reported to cause exacerbation of psychosis, and may also interact with antipsychotic and antidepressant medications. However, other workers have reported its use without problem, and to good effect (Kofoed et al, 1986). When used, it is probably best to maintain dose levels below $500 \mathrm{mg}$ daily. A possible alternative is calcium carbamide (Abstem), which remains available on a named-patient basis. Methadone maintenance or reduction programmes may be appropriate for opiate dependent patients - indeed claims have been made for an antipsychotic potential for opiates - but its use may alter neuroleptic dose requirements (Siris, 1990).

\section{Conclusions}

Substance misuse among the severely mentally ill is a common, and probably increasing problem. 
Whether drugs can cause prolonged and independent psychotic states, or whether they precipitate psychosis in vulnerable individuals is perhaps less important in clinical practice than their clearly detrimental effect on the course and prognosis of mental illness. These patients are often on the margins of psychiatric services, alienated by their poor compliance, social instability, and behavioural problems, and by the inflexibility of service structures to accommodate them. Their care demands an integration of skills, and a recognition of the slow timescale necessary for change. The greatest impediment to care, however, is often the lack of awareness of substance misuse and its underdiagnosis.

\section{References}

Ananth, J., Vandewater, S., Kamal, M., et al (1989) Missed diagnosis of substance abuse in psychiatric patients. Hospital and Community Psychiatry, 40, 297-299.

Andreasson, S., Allebeck, P., Engstrom, A., et al (1987) Cannabis and schizophrenia: a longitudinal study of Swedish conscripts. Lancet, ii, 1483-1486.

Arndt, S., Tyrrell, G., Flaum, M., et al (1992) Comorbidity of substance abuse and schizophrenia: the role of pre-morbid adjustment. Psychological Medicine, 22, 379-388.

Bartels, S. J., Teague, G. B., Drake, R. E., et al (1993) Substance abuse in schizophrenia: service utilization and costs. Journal of Nervous and Mental Diseases, 181, 227-232.

Black, D. W., Winokur, G., Bell, S., et al (1988) Complicated mania: comorbidity and immediate outcome in the treatment of mania. Archives of General Psychiatry, 45, 232-236.

Bowers, M. B., Mazure, C. M., Nelson, J. C., et al (1990) Psychotogenic drug use and neuroleptic response. Schizophrenia Bulletin, 16, 81-85.

Breakey, W. R., Goodell, H., Lorenz, P. C., et al (1974) Hallucinogenic drugs as precipitants of schizophrenia Psychological Medicine, 4, 255-261.

Buckley, P., Thompson, P., Way, L., et al (1994) Substance abuse among patients with treatment-resistant schizophrenia: characteristics and implications for clozapine therapy. American Journal of Psychiatry, 151, 385-389.

Chick, J., Badawy, A. \& Borg, S. (1993) Identification of excessive drinking and alcohol problems. Alcohol and Alcoholism, (suppl. 2), 121-125.

Cuffel, B. J. (1992) Prevalence estimates of substance abuse in schizophrenia and their correlates. Journal of Nervous and Mental Diseases, 180, 589-592.

-, Shumway, M., Chouljian, T. L., et al (1994) A longitudinal study of substance use and community violence in schizophrenia. Journal of Nervous and Mental Diseases, 182, 704-708.

Dixon, L., Haas, G., Weiden, P. J., et al (1991) Drug abuse in schizophrenic patients: clinical correlates and reasons for use. American Journal of Psychiatry, 148, 224-230.

Drake, R. E. \& Wallach, M. A. (1989) Substance abuse among the chronic mentally ill. Hospital and Community Psychiatry, 40, 1041-1046.

—, McHugo, G. J. \& Noordsy, D. L. (1993) A pilot study of outpatient treatment of alcoholism in schizophrenia: fouryear outcomes. American Journal of Psychiatry, 150, 328-329.

Duke, P. J., Pantelis, C. \& Barnes, T. R. E. (1994) South Westminster schizophrenia survey: alcohol use and its relationship to symptoms, tardive dyskinesia and illness onset. British Journal of Psychiatry, 164, 630-636.

Glass, I. B. (1989) Alcoholic hallucinosis: a psychiatric enigma - 1. The development of an idea. British Journal of Addiction,
84, 29-41.

Jablensky, A., Sartorius, N., Ernberg, G., et al (1992) Schizophrenia: manifestations, incidence and course in different cultures. Psychological Medicine (suppl. 20), 79.

Kofoed, L., Kania, J., Walsh, T., et al (1986) Outpatient treatment of patients with substance abuse and coexisting psychiatric disorders. American Journal of Psychiatry, 143, 867-872.

Lehman, A. F., Myers, C. P. \& Corty, E. (1989) Assessment and classification of patients with psychiatric and substance abuse syndromes. Hospital and Community Psychiatry, 40, 1019-1025.

Linszen, D. H., Dingemans, P. M. \& Lenior, M. E. (1994) Cannabis abuse and the course of recent-onset schizophrenic disorders. Archives of General Psychiatry, 51, 273-279.

Martinez-Arevalo, M. J., Calcedo-Ordonez, A. \& Varo-Prieto, J. R. (1994) Cannabis consumption as a prognostic factor in schizophrenia. British Journal of Psychiatry, 164, 679-681.

McGuire, P. K., Jones, P., Harvey, I., et al (1995) Morbid risk of schizophrenia for relatives of patients with cannabisassociated psychosis. Schizophrenia Research, 15, 277-281.

Regier, D. A., Farmer, M. E., Rae, D. S., et al (1990) Comorbidity of mental disorders with alcohol and other drug abuse: results from the Epidemiological Catchment Area (ECA) study. Journal of the American Medical Association, 264, 25112518.

Salloum, I. M., Moss, H. B. \& Daley, D. C. (1991) Substance abuse and schizophrenia: impediments to optimal care. American Journal of Drug and Alcohol Abuse, 17, 321-336.

Schneier, F. R. \& Siris, S. G. (1987) A review of psychoactive substance use and abuse in schizophrenia: patterns of drug choice. Journal of Nervous and Mental Diseases, 175, 641-652.

Selzer, J. A. \& Lieberman, J. A. (1993) Schizophrenia and substance abuse. Psychiatric Clinics of North America, 16, 401412.

Siris, S. G. (1990) Pharmacological treatment of substanceabusing schizophrenic patients. Schizophrenia Bulletin, 16, 111-122.

Smith, J. \& Hucker, S. (1994) Schizophrenia and substance abuse. British Journal of Psychiatry, 165, 13-21.

Strakowski, S. M., Tohen, M., Stoll, A. L., et al (1993) Comorbidity in psychosis at first hospitalization. American Journal of Psychiatry, 150, 752-757.

Thomas, H. (1993) Psychiatric symptoms in cannabis users. British Journal of Psychiatry, 163, 141-149.

\section{Multiple choice questions}

1 Substance misuse in schizophrenia:

a occurs in less than $10 \%$ of patients

b is associated with a later age of onset of psychosis

c more commonly takes the form of stimulant use than opiate use

d may be associated with better premorbid psychosocial adjustment

e results in increased relapse

2 Cannabis use:

a may precipitate paranoid symptoms in previously well individuals

$\mathrm{b}$ is clearly associated with a prolonged 'cannabis psychosis'

c is associated with better outcome in established schizophrenic illness

d worsens extrapyramidal side-effects of antipsychotics 
e is associated with earlier relapse of psychosis

3 Patients with chronic psychotic illnesses and substance misuse:

a comply poorly with treatment

b may respond less well to treatment even when complied with

c are at increased risk of suicide

$\mathrm{d}$ are readily diagnosed using urine drug screens

e are not treatable under provisions of the Mental Health Act

4 In the management of dual diagnosis patients:

a confrontational group meetings have been shown to be most effective

b good collaboration between general psychiatry and addiction specialities is essential c early achievement of abstinence is a prerequisite

d acute alcohol intake lowers neuroleptic bioavailability

e MAOIs are preferable in treating depressive disorders

MCQ answers

$\begin{array}{lllll}1 & 2 & 3 & 4\end{array}$

a $F$ a $T$ a $T$ a $F$

b $F$ b F b $T$ b $T$

c $T$ c $F$ c $T$ c $F$

d $T$ d F d F d F

e $T$ e $T$ e $F$ e $F$ 